\title{
Virtual Laboratories for a Course on Indoor Environmental Quality
}

\author{
doi:10.3991/ijoe.v5s2.1107 \\ M. Gameiro da Silva \\ University of Coimbra, Coimbra, Portugal
}

\begin{abstract}
This paper describes a set of software tools developed by the author to support the teaching of a course on Indoor Environmental Quality, given to the Master programme on Energy for Sustainability and PhD programme on Sustainable Energy Systems at the University of Coimbra, which are included on the MITPortugal Initiative educational programme. The course includes materials about Thermal Environment, Indoor Air Quality, Noise, Vibration, Lighting and the combined effect of these previous stressors. The software tools were developed both with Microsoft Excel and the programming platform LabVIEW.
\end{abstract}

Index Terms-Indoor Air Quality, Thermal Comfort, Noise measurements, Teaching Software Tools, Virtual Laboratories

\section{INTRODUCTION}

The author teaches a course on Indoor Environmental Quality in the Master and PhD programs on Energy for Sustainability at the University of Coimbra, Portugal. In the teaching methodology of this course there is a coexistence of the different approaches commonly used to deal with engineering problems, i.e. analytical formulation, experimentation and numerical simulation. The main objective is to demonstrate concepts in a stimulating way and to provide effective teaching tools for students, improving their way of establishing connections between theory and practice; thus enhancing their physical sense of reality. Various software tools related with indoor environmental quality were developed, by different authors, many of them with the aim of facilitating the calculations required by some standards where computational methods are needed. References [1] and [2] presented a first tool running under MS-DOS, called TEE, to perform the calculations required by different standards about indoor thermal environment, both for comfortable (PMV and ET*) and heat stress conditions (WBGT, SWreq, IREQ). An evolution of this tool was developed by [3], including new features and running under the MS-Windows system. Some excel spreadsheets for the calculation of the PPD and PMV indices were written by [4]. The latest version is available on the web site of an international company that manufactures measurement systems dedicated to thermal environment evaluation. A Java applet tool developed by [5] to perform the calculations related to the ISO 11079 standard has also been available for some years on the internet. The latest version, after the recast of the standard, is 4.1 .
The main innovation introduced by the author on his tools, relatively to the existing ones, is the display of all the calculation process, allowing, for instance, the breakdown of the human body thermal balance, into all its components, which is very effective as a pedagogical tool. Some innovative teaching strategies used in this course were already reported in previous papers ([6]; [7]; [8]; [9]).

\section{SOFTWARE TOOLS}

A diversified set of software tools was developed to allow the simulation of physical phenomena, the implementation of calculation procedures and the emulation of measuring and sound generation equipment using a portable computer and its sound card.

As regards Thermal Environment, a set of spreadsheets was written in Microsoft Excel to calculate the PMV and PPD thermal comfort indices, allowing the breakdown of the different heat fluxes participating on the human body thermal balance. Various spreadsheets were written to take into account the different possibilities of measuring equipment sets used to evaluate the indoor climate conditions.

In the case of Indoor Air Quality, the developed tools consist on simulators of the time evolution of the concentration of pollutants in indoor compartments written in LabVIEW. The cases of metabolic CO2 and pollutants released by tobacco smoke were considered.

Three different virtual instruments were developed for the Acoustics part of the course - a Sound Pressure Level Meter, a Noise Equivalent Level Meter and a Third Octave Frequency Analyzer - using the sound card of a portable computer as data acquisition device.

Another application tool, consisting of a sound generator, was also developed. The sound card of the computer is also used to generate and add sinus sounds with frequencies centered on the octave bands between 63 and $8000 \mathrm{~Hz}$.

\section{A. Thermal Comfort indices PMV and PPD}

The method developed by [10] and adapted in the ISO Standard 7730 is based on the determination of the PMV index (Predicted Mean Vote), calculated from an equation of thermal balance for the human body (see Figure 1), involving the internal generation terms and heat exchanges with the surrounding environment. 


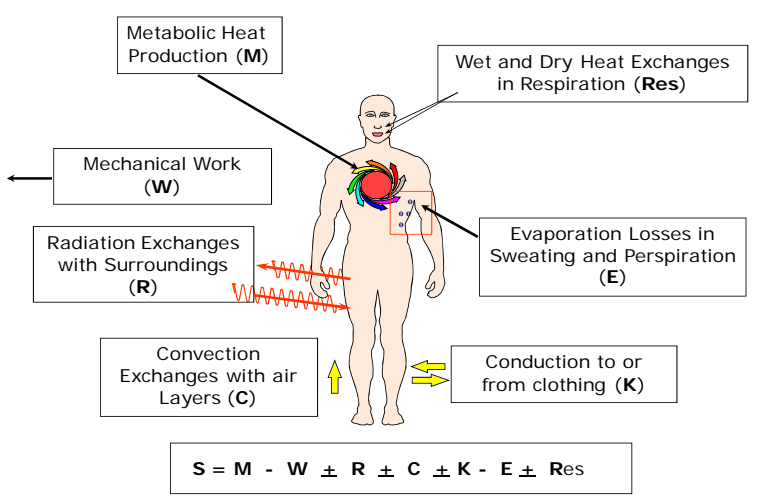

Figure 1. Human body thermal balance

A correlation model between the subjective human perception, expressed through the vote of comfort on a scale ranging from -3 (very cold) to +3 (very hot), and the difference between the heat generated and the heat released by the human body, was established, which corresponds to the following equation:

$$
\begin{aligned}
& P M V=\left(0,303 e^{-2,1 M}+0,028\right) \times \\
& \times\left[(M-W)-H-E_{C}-C_{r e s}-E_{r e s}\right]
\end{aligned}
$$

where the different terms stand for, respectively:

$M$ - metabolic rate $\left(\mathrm{W} / \mathrm{m}^{2}\right)$;

$W$ - effective mechanical power $\left(\mathrm{W} / \mathrm{m}^{2}\right)$;

$H$ - sensitive heat losses;

$E_{c}$ - heat exchange by evaporation on the skin;

$C_{\text {res }}$ - heat exchange by convection in breathing;

$E_{\text {res }}$ - evaporative heat exchange in breathing.

The set of equations for the implementation of the method is given in ISO 7730 [11]. The main problem in the calculation results from the fact that the term corresponding to the external temperature of clothing is unknown, a priori. This temperature must be determined by an iterative process, from an equation resulting from the heat balance established for the clothing layer. It is considered that, in a steady-state regime, the heat flux transmitted by conduction through that same clothing from the inner layer, at skin temperature, until the outer layer, is equal to the sum of the heat exchanges by convection and by radiation with the surrounding environment.

The other index proposed in the ISO Standard 7730 is PPD (Predicted Percentage of Dissatisfied) that quantifies the expected percentage of dissatisfied people in a given thermal environment.

The variation of the PPD index, as a function of PMV, can be approximated by an analytic expression that corresponds to a curve whose appearance is similar to an inverted Gaussian distribution:

$$
P P D=100-95 \times e^{-\left(0,00353 \times P M V^{4}-0,2179 \times P M V^{2}\right)}
$$

The first developed spreadsheet directly applies the method recommended by the ISO Standard 7730, determining the comfort indices based on the following input data related to environmental conditions - air temperature, mean radiant temperature, air velocity and partial pressure of vapour. In the graphical interface of the spreadsheet (see Figure 2) and in addition to the comfort indices, the values of the various heat fluxes in certain intermediate calculations are presented, which helps to develop the physical sensitivity of the user to this topic.

The spreadsheet, of which a graphical interface for a given instance is presented in Figure 2, is divided into three sections: the first, located on the left side, is used for data input by the operator and the initiation and control of the calculation process; the second, in the middle, displays the results of intermediate calculations required to implement the method and, finally, the third section, located on the right side of the screen, where the output values of the two comfort indices, PMV and PPD, are presented.

For each situation, the user has to fill in the seven cells in the field of data entry on the top left corner, which are the only cells throughout the spreadsheet that allow input from the user, and then click on the button named "Run", which starts the calculation process that lasts less than one second.

The spreadsheet also provides, as output, a graphical representation of the relative weights, in percentage, of the different parts of heat exchange fluxes between the individual and the surrounding environment. Figure 3 shows the graph obtained for the situation corresponding to the data in Figure 2.

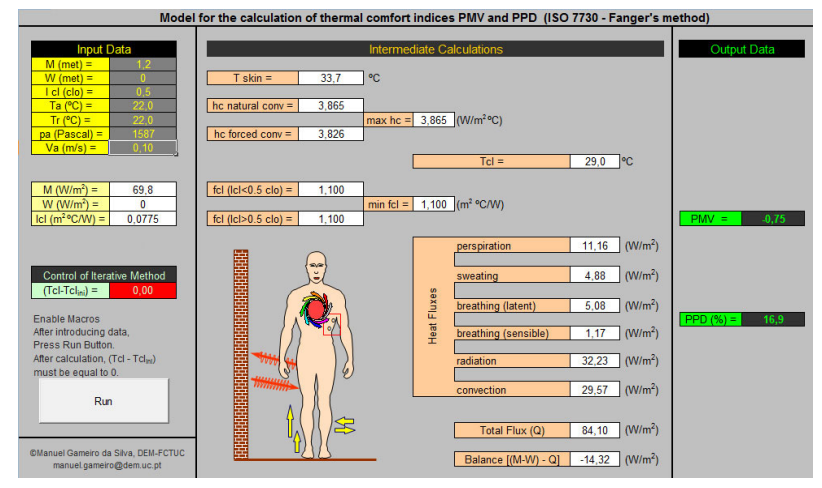

Figure 2. Graphical interface of the basic spreadsheet for PMV and PPD calculation

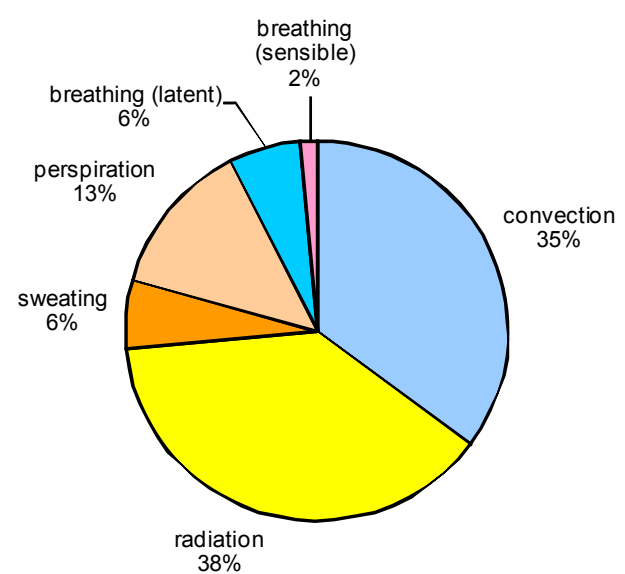

Figure 3. Breakdown of heat and mass fluxes for the situation presented in figure 2 . 
Apart from the basic spreadsheet previously presented, some more versions were developed to ensure determination of PMV and PPD indices in situations where measurements were made with sets of sensors that, although not measuring directly the four environmental parameters $\left(\mathrm{t}_{\mathrm{a}}, \mathrm{t}_{\mathrm{r}}, \mathrm{p}_{\mathrm{a}}\right.$ and $\left.\mathrm{v}_{\mathrm{ar}}\right)$, allow their calculation.

\section{B. Indoor Air Quality Simulation Tools - General equations for the time evolution of a pollutant concentration}

Consider a uni-zone compartment, where there is a source of pollution that has gas exchanges with the outside air, and where an air purifier may work. Admitting the possibility of deposition and absorption of the pollutant on the walls and other surfaces, the temporal evolution of the concentration of a pollutant is modeled by the following differential equation:

$$
\frac{d C}{d t}=\frac{G}{V}+\lambda_{v} C_{e x t}-\lambda_{v} C-v \frac{S}{C}-\frac{Q_{a c}}{V} C \varepsilon_{a c}
$$

with:

$C$, instantaneous average concentration of pollutant $\left(\mathrm{mg} / \mathrm{m}^{3}\right)$

$G$, pollutant generation inside the compartment $(\mathrm{mg} / \mathrm{h})$

$V$, room volume $\left(\mathrm{m}^{3}\right)$

$\lambda_{v}$, air exchange rate (h-1), i.e., fresh air flow-rate divided by the room volume

$C_{\text {ext }}$, concentration of the pollutant in outside air $\left(\mathrm{mg} / \mathrm{m}^{3}\right)$

$v_{d}$, rate of deposition of the pollutant $\left(\mathrm{mg} / \mathrm{h} \cdot \mathrm{m}^{2}\right)$

$S$, surface of deposition $\left(\mathrm{m}^{2}\right)$

$Q_{a c}$, flow rate through the air purifier $\left(\mathrm{m}^{3} / \mathrm{h}\right)$

$\varepsilon_{a c}$, efficiency of the air purifier (dimensionless).

The effects of absorption or deposition of the pollutant inside the compartment and the removal by filtering through the air purifier system may be considered in a simplified form, reducing the intensity of the source. Thus, for purposes of simplification, their terms in the equation may be neglected, giving:

$$
\frac{d C}{d t}=\frac{G}{V}+\lambda_{v} C_{e x t}-\lambda_{v} C(t)
$$

that, integrated for a situation where $V, G, C_{e x t}$ and $Q$ remain constant, from an initial time instant $t=0$, where the initial concentration $C_{0}=C_{e x t}$, till a generic time instant $t$, will lead to:

$$
C(t)=C_{\text {equi }}+\left(C_{0}-C_{\text {equi }}\right) \times e^{-\lambda_{v} t}
$$

The equilibrium concentration $C_{\text {equi }}$, in the equation above, is the value that occurs when the steady-state regime is reached, i.e. when the variation in concentration ceases. Thus, it is obtained equating the first member of equation 4 (the time derivative of the concentration) to zero, which gives:

$$
C_{e q u i}=C_{e x t}+\frac{G}{V \times \lambda_{v}}
$$

that, with $\lambda_{v}=Q / V$, becomes:

$$
C_{\text {equi }}=C_{\text {ext }}+\frac{G}{Q}
$$

The implemented numerical method is based on an approximation of equation (4), through the trapezoidal rule, which becomes:

$$
\Delta C=\left[\frac{G}{V}+\lambda_{v} C_{e x t}-\lambda_{v} C(t)\right] \times \Delta t
$$

Once the size of the step interval $(\Delta t)$ for the calculations has been fixed, the method is numerically implemented within a "While" loop where the variation of concentration $(\Delta C)$ during the interval is calculated for each step and added to the value occurring after the previous step $\left(\mathrm{C}_{\mathrm{i}-1}\right)$. The values of the room volume $\mathrm{V}$, of the initial concentration of pollutant in outside air $C_{i n i}$, and of the step interval $\Delta t$ are defined at the beginning of the simulation and remain constant till the end of the run. The intensity of the source $(\mathrm{G})$ and the fresh air flow-rate (Q) may be changed while the program is running, allowing a visualization of the effects of their changes.

The outputs of the simulation are the instantaneous concentration of the pollutant, the graphical display of the time-evolution of the pollutant concentration and, once the simulation has been stopped by the operator, the statistical data about the time series of concentration values (average, maximum, minimum and standard deviation).

\section{Metabolic $\mathrm{CO}_{2}$ simulation tool}

In [12], the expressions to calculate the volumes of Oxygen $\left(\mathrm{O}_{2}\right)$ and Carbon Dioxide $\left(\mathrm{CO}_{2}\right)$ in the human respiration process are given as a function of the metabolic rate and the corpulence of the studied person. The volume of consumed $\mathrm{O}_{2}$ is given by:

$$
V_{O_{2}}=\frac{0,0027 \cdot A_{D} \cdot M}{0,23 \cdot R Q+0,77} \quad 1 / \mathrm{s}
$$

where $A_{D}\left(\mathrm{~m}^{2}\right)$, the DuBois area, is the body skin area of the analyzed person, $\mathrm{M}$ is the metabolic rate expressed in met, with 1 met $=58.15 \mathrm{~W} / \mathrm{m}^{2}$ and $\mathrm{RQ}$ is the ratio between the volume of released $\mathrm{CO}_{2}$ and the consumed volume of $\mathrm{O}_{2}$. RQ usually takes the value 0.83 , but it may vary till 1 , for a person with a very high metabolic rate (more than $5 \mathrm{met}$ ). $\mathrm{A}_{\mathrm{D}}$ is calculated from:

$$
A_{D}=0,203 \times\left(H^{0,725}\right) \times\left(W^{0,425}\right)
$$

where $\mathrm{H}(\mathrm{m})$ and $\mathrm{W}(\mathrm{kg})$ are, respectively, the height and the weight of the analyzed person. Once the volume of $\mathrm{O}_{2}$ has been calculated, the volume of released $\mathrm{CO}_{2}$, for normal metabolic rate cases is computed from: 


$$
V_{\mathrm{CO}_{2}}=R Q \times V_{\mathrm{O}_{2}}
$$

In figure 4 it is depicted the front panel of the software, after a test performed for a one day evolution in an office, occupied from 08:30 till 18:00 by one person. It was assumed that the person has a metabolic rate of $1.2 \mathrm{met}$ $\left(69.78 \mathrm{~W} / \mathrm{m}^{2}\right)$ when at the office, a typical value for office work. The considered weight and height are for a person close to the European percentile 50. A daily routine with two breaks, a short one in the middle of the morning and about one hour at lunch time was simulated.

\section{Simulation tool for PM10 released by tobacco}

A similar application was implemented to simulate the Particulate Matter with diameter below 10 micron (PM10) released by tobacco cigarettes and pipes, because this type of pollutant is the one that poses more problems in terms of needed fresh air flow-rate to comply with limits for pollutants recommended for IAQ.

It is possible to regulate the rate of particle release by the cigarette and to light or extinguish it during the simulation.

Typical values of pollutants usually checked in IAQ audits and released by one cigarette burning are given in TABLE 1, which summarizes information collected in [13] and [14]

TABLE I.

TyPICAL POLLUTANT QUANTITIES ANALYZED IN IAQ AUdiTS For ONE CigARETTE

\begin{tabular}{|l|l|l|}
\hline Type & \multicolumn{1}{|c|}{ Pollutant } & Unit (mg) \\
\hline Particles & Particles suspended in air (PM10) & $18^{(1)}$ \\
\hline \multirow{4}{*}{ Chemical } & Carbon Dioxide $\left(\mathrm{CO}_{2}\right)$ & $160-550^{(2)}$ \\
\cline { 2 - 3 } & Carbon Monoxide (CO) & $60^{(1)}$ \\
\cline { 2 - 3 } & Formaldehyde (HCHO) & $0,4^{(2)}$ \\
\cline { 2 - 3 } & Total Volatile Organic Compounds & 3,6 \\
\hline
\end{tabular}

In figure 5 is presented the software front panel after a simulation of a two hour period in a room occupied by a smoker. The fresh air flow-rate was fixed at $60 \mathrm{~m} 3 / \mathrm{h}$, because this is the recommended value in the Portuguese legislation for compartments with smokers in new buildings. A situation was simulated where four cigarettes were smoked during the two hours. The average spatial concentration of PM10 in the room exceeds clearly the recommended limits for indoor spaces.

\section{E. Sound Measuring Virtual Instruments}

Three different virtual instruments were developed - a Sound Pressure Level Meter, a Noise Equivalent Level Meter and Recorder and a Third Octave Frequency Analyzer. The software application tools were written in the programming platform LabVIEW 7.1, using some of the Noise and Vibration Toolkits pertaining to the software package. The found solution, as regards the sound signal data acquisition, is very convenient because the computer sound card is used, which results in a very low investment. For teaching purposes, where the objective is mainly to demonstrate concepts and accuracy requirements may be lower than in the case of legal metrology measurements, a sound card with a 16 bits AD converter is enough. A stereo electret condenser microphone SONY DS70P was plugged to the computer sound card and used as primary sensor of the measuring chain.

In the design of the graphical interfaces of the virtual sound measuring instruments, the idea was to reproduce the same type of controls for setting parameters as those existing in real measuring devices. As regards data displays, the objective was to be more elucidative than in real instruments, allowing the simultaneous display of the different phases of signal processing, from the beginning till the end.

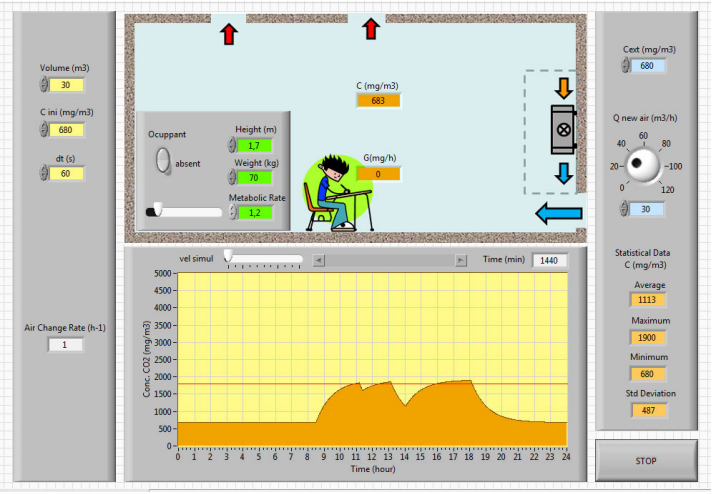

Figure 4. Front panel of the simulation tool for metabolic $\mathrm{CO}_{2}$ concentration after a one day simulation for an office

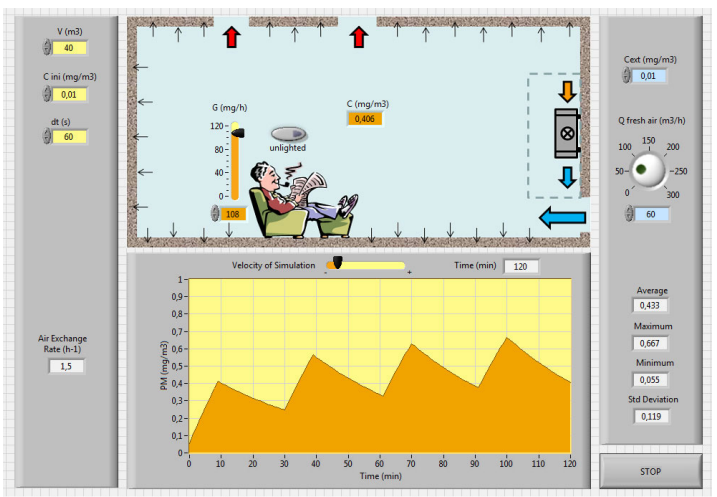

Figure 5. Front panel of the simulation tool for particulate matter released by tobacco

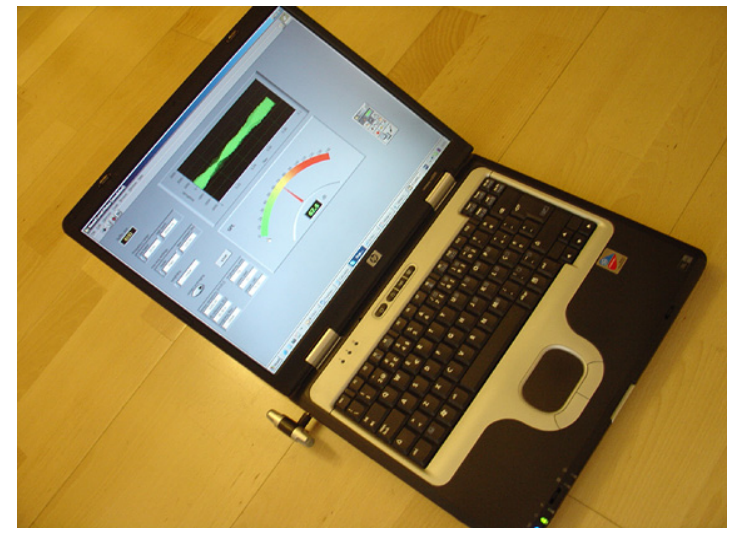

Figure 6. Computer with microphone running the SPL meter emulator software 
Digital displays were used to update the value of the main sound descriptors while the measurements are running. Students were very enthusiastic using these virtual instruments because they can see in real time the effect of a different sound in the displays. Interaction with the software is very easy because the users themselves can produce different sounds and look at their effect on the virtual instruments.

The first software tool that was developed emulates a very simple soundmeter. Its graphical interface is presented in Fig. 8. It calculates, from a time series with the values of pressure measured by the microphone in the room, the sound pressure (SP) value, i.e. the standard deviation of pressure, and the sound pressure level, in $\mathrm{dB}$, defined as:

$$
S P L=20 \times \log \frac{S P}{S P_{r e f}}
$$

where $\mathrm{SP}_{\text {ref }}$ is the threshold of hearing $(20 \mu \mathrm{Pa})$.

Besides displaying in digital and analog formats the value of the Sound Pressure Level, the virtual instrument has a scopemeter where the time evolution of the pressure signal measured by the microphone is updated. In this way, students can have a better understanding of the relationships between the sound signal and its descriptors. It is possible to adjust some parameters, as it happens in a real soundmeter, e.g. the weighting curve (Linear, A, B, $\mathrm{C}$ or $\mathrm{D})$, the frequency range, the amplification gain, etc. The sensitivity of the microphone, in the control panel of the software, is adjusted using a constant noise level source and a calibrated soundmeter as reference. With the microphone connected to the computer and the soundmeter's microphone placed in equivalent positions relatively to the noise source, the sensitivity of the microphone is adjusted in order to get the same reading in both systems. This adjustment is dependent on the setting of the microphone gain that has been defined for the microphone channel in the sound card setting software. In computers working with Microsoft Windows operating system this adjustment is defined in Control Panel 1 Sound and Audio Devices Properties. In case it is changed, the sensitivity of the microphone in the virtual soundmeter software should be adjusted.

When analyzing the effect of sound over a certain period of time, there is the need for another type of descriptor. The most used is the Noise Equivalent Level (Leq), which is the Sound Pressure Level of a sound with constant amplitude with the same duration and energy content of the real signal which is being analyzed.

The following formula is used to calculate Leq from a time series of sound pressure values:

$$
L_{e q}=10 \times \log \frac{1}{n} \sum_{1}^{n} 10^{\frac{S P L_{i}}{10}}
$$

The noise equivalent level is sometimes referred as the energetic mean of the sound pressure level time series and it is in general far from the value of the arithmetic mean, because maxima periods of SPL weight much more in its calculation than minima periods.

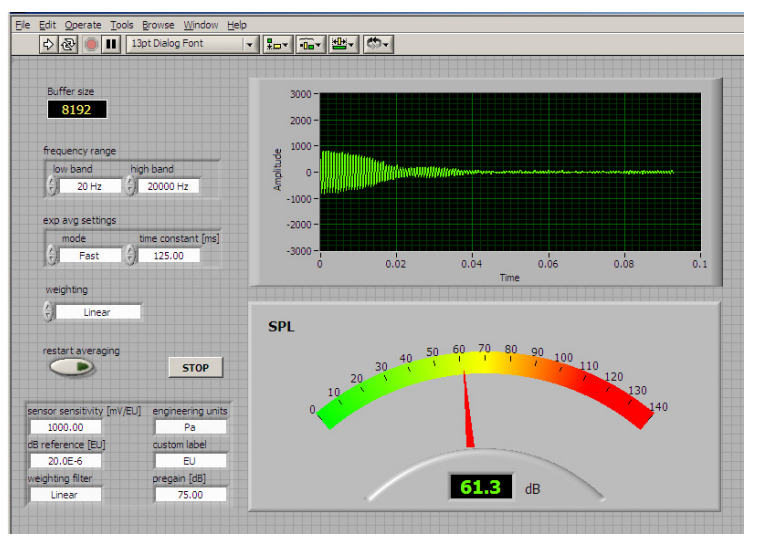

Figure 7. Graphical interface of the SPL meter emulator software

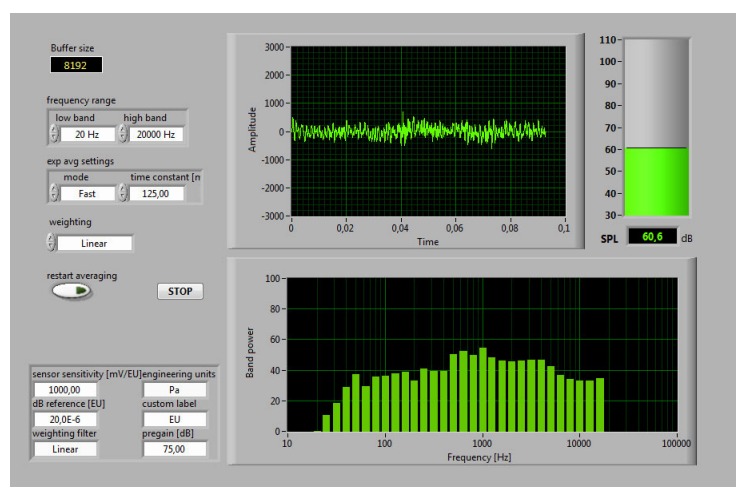

Figure 8. Graphical interface of the Third Octave Frequency Analyzer software

The second software tool that was developed, to measure and display graphically the time evolutions of SPL and Leq is very convenient to call the attention of students for this fact and consequently to the special care that must be taken when defining sampling parameters for sound signals.

In Fig. 8 the graphical interface of the Leq meter and recorder virtual instrument software is displayed. A scopemeter with the time evolution of the pressure fluctuations measured by the microphone during the last 0.1 seconds is displayed on the top of the screen, being the computed Sound Pressure Level updated in the upper right part both on a digital display and a bar graph. In the graph on the lower part of the screen the time evolutions of SPL and Leq values are displayed. While the graph of SPL is updated with the value calculated for the last interval, the value of Leq corresponds to the numerical integration from the beginning of the measurement. Analyzing the graphs of SPL (green) and Leq (yellow) displayed in figure 9, the characteristics of Leq evolution are patent. Once a certain level is reached it takes a very long time to have a significant reduction even if the SPL values remain at a low level.

There is a button to restart the averaging process in the left part of the screen, as well as controls to set the measurement parameters of the emulated soundmeter, as regards the frequency range, the weighting factors, the sampling time for SPL calculation and the sensitivity of the sound measuring hardware (microphone, preamplifier, etc). 


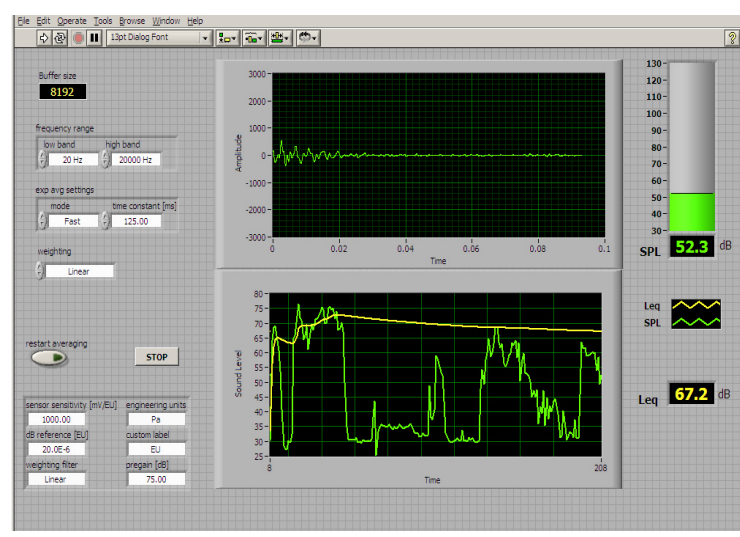

Figure 9. Graphical interface of the Leq meter emulator software

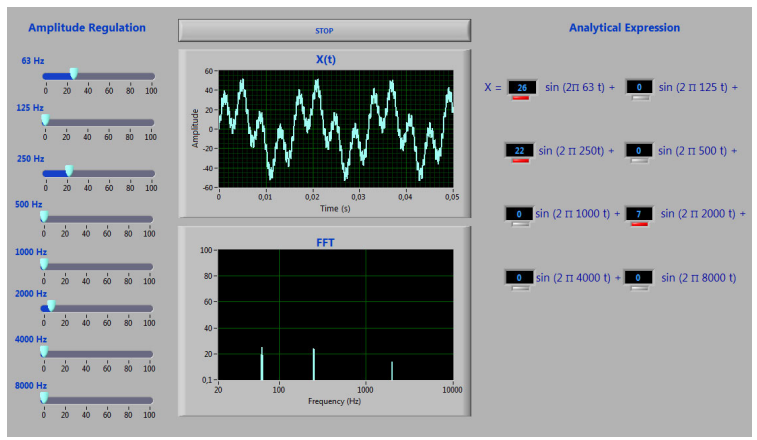

Figure 10. Graphical interface of the sound generator software

The third emulated virtual instrument is a third-octave band frequency analyzer. It is based on a Fast Fourier Transform algorithm and allows the real time display of the frequency analysis of the acquired sound signal.

The signal is decomposed into 30 elementary signals, each one of them with a frequency and amplitude given by the respective bar in the frequency spectrum graph. The real time display of the frequency spectrum of the signal helps students to feel the relationship between the graph and the respective Fourier series and the tonal content of the heard sound. In order to cement the concepts about the Fourier series and also the sum of sound pressure levels from various sources, students are asked to calculate the overall SPL using the information given in the Power Spectrum. This calculation is to be performed with and without the application of the frequency weighting curves.

\section{F. Sound Generator}

Also based upon the computer sound card, another software tool, similar to a simple synthesizer, was developed to allow the generation and reproduction of sounds, through the addition of mono-tonal sinus sound waves with eight possible frequencies, corresponding to the core values of the octave bands ranging from $63 \mathrm{~Hz}$ to $8 \mathrm{kHz}$.

The software, whose graphical interface is presented in Figure 10, allows the regulation of the amplitudes of sounds in the eight octave bands through the set of sliders located on the left side. In the center of the screen are located two graphical displays, being displayed on the upper one the time evolution of the generated signal and on the lower one the results of the application of the Fast Fourier Transform to the generated signal, with the identification of the elementary frequencies and respective amplitudes.

On the right side, the analytical expression of the Fourier series corresponding to the generated signal is displayed, which is simultaneously graphically presented and played through the loudspeakers of the computer. In the analytical expression, below the digital displays which are showing the amplitudes for each frequency, there are LED-type indicators shifting from grey to red if the amplitude value scale is different from zero, allowing for a rapid identification of what are the terms of the expression that are not null.

\section{CONCLUSION}

The use of software tools, both for the modeling of physical processes and for the acquisition of data from experimental setups, resulted in a sound increase of the enthusiasm of the learners for the matters lectured in the course of Indoor Environmental Quality. They were used for the explanation of materials during lessons, as the basis for practical exercises and also tested by students during some homework exercises. The tools were made available to the students through the University of Coimbra Web on Campus platform. The excel spreadsheet for PMV and PPD calculations is permanently available to everybody and has been used by researchers both in Portugal and other countries. The comments received from users are very enthusiastic.

A first experience of an internet remote access was also demonstrated in the class room with the described virtual instruments, using the web publishing tool functionality of LabVIEW software. The laptop computer of the teacher was used as the server, its URL was given to the students and they could in their place follow the measuring procedure and gain control of it. In the near future, the described virtual instruments will be integrated in a set of remote access laboratories that is now under implementation in the Department of Mechanical Engineering of the University of Coimbra.

\section{REFERENCES}

[1] Alfano, G., Cesarano, G. and d'Ambrosio, F.R., 1991. A computer program for thermal environment evaluation, Proceedings of the 11th Congress of the International Ergonomics Association pp. 924-926.

[2] Alfano G., d'Ambrosio F.R., Riccio G., 1992. An evaluation of thermal environments also for non specialist. Proceedings of 5th International Conference on Environmental Ergonomics, Maastricht, pp. 84-85.

[3] d'Ambrosio Alfano, F.R., Palella, B.I., Riccio, G., 2004. A friendly tool for the assessment of thermal environments, Climamed 2004, 16-17 April 2004, Lisbon

[4] Nilsson, H., 2005, PMVcalc_v2_English.xls - PMV and PPD calculation, Department of Technology and Built Environment, Lab. of Ventilation and Air Quality, University of Gavle,

[5] available at http://www.lumasenseinc.com/pmv_calculation.html, accessed 20-09-2009

[6] Nilsson, H. and Holmer, I., 2007. IREQ 2007 ver 4.1, - JAVA applet for ISO 11079 - Calculation of Required Clothing Insulation (IREQ), Duration Limited Exposure (Dlim), Required Recovery Time (RT) and Wind Chill Temperature (twc)

[7] Available at http://wwwold.eat.lth.se/Forskning/Termisk/Termisk_HP/Klimatfi ler/IREQ2002alfa.htm, accessed 21-09-2009

[8] Gameiro da Silva, M.C., 2007. Sound Measuring Virtual Instruments, International Conference on Remote and Virtual Laboratories (REV 2007), Porto, 23-27 June 2007. 


\section{VIRTUAL LABORATORIES FOR A COURSE ON INDOOR ENVIRONMENTAL QUALITY}

[9] Gameiro da Silva, M.C., 2008. “ Aplicações Computacionais para Avaliação do Conforto Térmico, Revista Climatização, $\mathrm{n}^{\circ} 56$, Ano VIII, Março/Abril 2008, pp. 56-68.

[10] Gameiro da Silva, M.C., Mateus, M.L.O.S., 2008., "Desenvolvimento de um conjunto de aplicações computacionais para emulação de equipamentos de medição e análise sonora", $\mathrm{V}$ Congresso Ibérico de Acústica, Accoustics European Symposium, 20-22 October 2008, Coimbra, Portugal.

[11] Gameiro da Silva, M.C., 2009. "Indoor Air Quality Simulation Tools", 5th CLIMAMED - HVAC\&R Mediterranean Congress, 16-18 April 2009, Lisboa, Portugal.

[12] Fanger, P.O, 1972. "Thermal Comfort", Danish Technical Press.

[13] ISO 7730, 2005. "Moderate Thermal Environments Determination of the PMV and PPD Indices and the Specifications of the Conditions for Thermal Comfort" - International Standard Organization, Genève, Suisse. (doi:10.1021/es072062w)

[14] Emmerich, S.J. and Persily, A.K., 2001. State-of-the-Art Review of $\mathrm{CO} 2$ Demand Controlled Ventilation Technology and Application, NISTIR 6729, National Institute of Standards and Technology, USA.
[15] Simone Charles et al., 2008. VOC and Particles Emissions from Commercial Cigarettes: Analysis of 2,5 DMF as an ETS Tracer, Environm. Sci. Technol. 2008, 42, 1324-1331.

[16] Ventilation and Smoking, 2004. REHVA GUIDE BOOK $n^{\circ} 4$, Hakon Skistad \& Ben Bronsema editors.

\section{AUTHORS}

Manuel Gameiro da Silva is with ADAI - LAETA, Department of Mechanical Engineering, University of Coimbra, Portugal, (e-mail: manuel.gameiro@dem.uc.pt)

This work was supported in part by a project envisaging the creation of Virtual and Remote Access Laboratories in the Department of Mechanical Engineering of the Faculty of Science and Technology of the University of Coimbra, which was granted by Banco Português de Investimento (BPI), during the academic year 2007/2008.

This article was modified from a presentation at the IRF'2009 conference in Porto, Portugal, July 2009. Submitted 16 October 2009. Published as resubmitted by the authors on 20 October 2009. 